\title{
Survival of Ixodes ricinus (Acari: Ixodidae) Under Challenging Conditions of Temperature and Humidity Is Influenced by Borrelia burgdorferi sensu lato Infection
}

\author{
CORALIE HERRMANN AND LISE GERN ${ }^{1}$ \\ Institute of Biology, Laboratory of Eco-Epidemiology of Parasites, University of Neuchâtel, Emile Argand 11, \\ Case postale 158, 2009 Neuchâtel, Switzerland
}

\begin{abstract}
J. Med. Entomol. 47(6): 1196-1204 (2010); DOI: 10.1603/ME10111
ABSTRACT To determine whether Borrelia burgdorferi sensu lato (s.l.) influences tick survival under thermohygrometric stress, Ixodes ricinus (L.) (Acari: Ixodidae) questing ticks were tested under various relative humidities $(13,32,51.5,61$, and $89 \% \mathrm{RH})$ at two different temperatures $\left(12.5\right.$ and $\left.25^{\circ} \mathrm{C}\right)$ and investigated for Borrelia infection. Survival rate of females was highest (77.6\%), followed by males $(51.6 \%)$, and nymphs $(43.2 \%)$. The thermohygrometric factor that most importantly determined survival was saturation deficit (SD). As SD increased, tick survival rate decreased in all stages. Among the 1,500 ticks tested for B. burgdorferi s.1., 34.8\% $(n=522)$ were infected. Adult infection rate $(39.6 \%)$ was higher than that of nymphs $(25.5 \%)$. Infection load in real-time polymerase chain reaction ranged from 1 to 1.2 million spirochetes per tick. B. afzelii (39.7\%), B. burgdorferi sensu stricto (12.1\%), B. garinii $(37.9 \%)$, B. myamotoi $(3.6 \%)$, and B. valaisiana $(23.8 \%)$ were recorded. B. garinii infected significantly less nymphs than adults whereas $B$. afzelii displayed the opposite trend. Survival rate of nymphal and adult $I$. ricinus was significantly enhanced by infection by B. burgdorferi s.l. ( $\chi^{2}:$ nymph, $P=0.008$; adult, $P=0.021)$. In adults, a negative effect of infection on tick survival was observed when spirochete load overcame a threshold estimated at 160,000 spirochetes per tick but not in nymphs. Moreover, ticks infected by B. afzelii survived better than other ticks (infected by other genospecies or not). The results here indicate that infection by B. burgdorferi s.l., and more specifically infection by B. afzelii, confers survival advantages to I. ricinus under challenging thermohygrometric conditions.
\end{abstract}

KEY WORDS Lyme disease, Ixodes ricinus, climate change, tick survival, Switzerland

The hard-bodied tick Ixodes ricinus (L.) (Acari: Ixodidae) is the main vector of Borrelia burgdorferi sensu lato (s.l.), the etiological agent of the most common tick-borne disease in Europe, Lyme borreliosis. Ten Borrelia genospecies have been described in $I$. ricinus in Europe: B. afzelii, B. bavariensis, B. bissettii, B. burgdorferi sensu stricto (s.s.), B. carolinensis, B. garinii, $B$. lusitaniae, B. spielmanii, B. valaisiana as well as a Borrelia species related to relapsing fever spirochetes, $B$. myamotoi (Rauter and Hartung 2005, Margos et al. 2009, Gern et al. 2010, Cotté et al. 2010). Both B. burgdorferi s.l. and ticks of the I. ricinus complex have been thoroughly studied on their own, but knowledge on the influence of infection by Borrelia on ticks remains scarce.

The effect of infection by B. burgdorferi s.l. on the behavior of Ixodes ticks has been documented. Hence, it was observed that infection by B. burgdorferi s.l. in I. persulcatus and I. ricinus ticks reduced motor activity of both adult and immature infected individuals compared with uninfected ticks (Alekseev et al. 2000). Moreover, Lefcort and Durden (1996) reported that

\footnotetext{
${ }^{1}$ Corresponding author, e-mail: lise.gern@unine.ch.
}

infection by B. burgdorferi s.l. influenced I. scapularis phototaxis, activity and questing height. Infected adults were less able to overcome physical obstacles, avoided vertical surfaces, were less active and quested at lower heights, whereas infected nymphs showed increased phototaxis and attraction to vertical surfaces. It also was observed that infection by B. burgdorferi s.l. increased questing activity in Ixodes persulcatus Schulze (Naumov 1999 cited by Uspensky et al. 2006) and host-finding efficacy in adult I. ricinus (Faulde and Robbins 2008).

In contrast, reports on the effect of parasites on the physiology of ticks are rare. Mather et al. (1993) observed a higher survival rate of Ixodes scapularis Say nymphs infected by B. burgdorferi s.l. than uninfected nymphs after habitat burning, suggesting that infection by B. burgdorferi s.l. makes ticks more resistant to heat and desiccation. Furthermore, in 1998 in Neuchâtel, Switzerland, a lower density of questing I. ricinus ticks was observed due to high desiccating climatic conditions (Perret et al. 2000) and an unexpectedly high percentage of nymphs harboring high numbers of $B$. burgdorferi s.l. was recorded compared with the previous and following 
years (L.G., unpublished data). In addition, Naumov (2003) (cited by Uspensky et al. 2006) noted that there was a trend toward an increase in the longevity of adult I. ricinus and I. persulcatus infected by B. burgdorferi s.l. spirochetes.

Similarly to other terrestrial arthropods, the biggest challenge for ticks is to maintain water balance while living in a relatively dry environment. This is even more crucial for I. ricinus survival because this tick is exceptionally sensitive to temperature and humidity compared with other ticks (Knülle and Rudolph 1982, Sonenshine 1991). Hence, combined with temperature, humidity is the most important limiting factor in I. ricinus survival and activity because the different developmental stages are known to display little resistance to desiccation (MacLeod 1935, Lees 1946, Aeschlimann 1972). In fact, relative humidity needs to be $>70-80 \%$ to allow questing tick activity and survival (MacLeod 1935). More precisely, saturation deficit (SD), a measure of the drying power of the atmosphere depending on both temperature and relative humdity (Randolph and Storey 1999), limits duration of questing (Perret et al. 2003, 2004) and survival of I. ricinus in nature (Perret 2002, Perret et al. 2000, 2004; Burri et al. 2007).

In the context of climate change and with observations of increasing values and longer lasting periods of high saturation deficits, as for example in the Neuchâtel region (Perret et al. 2000, Morán Cadenas et al. $2007 \mathrm{~b}$ ), it seemed necessary to study whether infection by B. burgdorferi s.l. may influence I. ricinus survival under thermohygrometric stress conditions.

\section{Materials and Methods}

Sampling of Questing Ticks. The sampling site was a mixed forest (deciduous dominant) situated at $600 \mathrm{~m}$ above sea level $\left(47^{\circ} 00^{\prime} \mathrm{N}, 6^{\circ} 57^{\prime} \mathrm{E}\right)$ on the south-facing slope of Chaumont Mountain (Neuchâtel, Switzerland). Host-seeking ticks were sampled by dragging a $1-\mathrm{m}^{2}$ terry flag over the low vegetation. The flag was examined for ticks every $10 \mathrm{~m}$. Questing nymphs and adults were collected on two different days in May 2009. They were held over water $(98 \% \mathrm{RH})$ at $4^{\circ} \mathrm{C}$ in the dark for rehydration for 4-6 wk (depending on testing day) as described in Crooks and Randolph (2006).

Fat Content Analysis. Fat content was quantified in field-collected and laboratory-reared ticks to take into account variable energy resources when assessing survival rate of $I$. ricinus under unfavorable thermohygrometric conditions. Samples of field-collected nymphs, males, and females were analyzed for their fat content as described in Randolph and Storey (1999) and as calculated in Crooks and Randolph (2006). As controls for nymphs, laboratory-reared nymphs from the colony at the University of Neuchâtel known to be free of spirochetal infection also were analyzed for their fat content. This colony is maintained at room temperature and natural light conditions.

Survival Tests. Survival arenas were made of 5-ml plastic culture tubes (Milian, Meyrin, Switzerland) sealed together by their screw caps with silicone Screw cap centers had been removed previously and replaced by polyamide mesh $(500-\mu \mathrm{m}$ grid, Sefar-Nitex, Sefar AG, Haiden, Switzerland). Arenas stood vertically, with the top culture tube containing ticks and the bottom tube filled two thirds with saturated salt solutions $\left(\mathrm{K}_{2} \mathrm{SO}_{4}, \mathrm{LiCl}, \mathrm{MgCl}_{2}\right.$, or $\left.\mathrm{NaBr}\right)$, producing known relative humditities at a given temperature based on Winston and Bates (1960). They were adjusted after 48-h tests conducted under experimental conditions.

Tick survival was studied under five thermohygrometric conditions: $25^{\circ} \mathrm{C}, 89 \% \mathrm{RH} ; 12.5^{\circ} \mathrm{C}, 61 \% \mathrm{RH}$ $12.5^{\circ} \mathrm{C}, 13 \% \mathrm{RH} ; 25^{\circ} \mathrm{C}, 51.5 \% \mathrm{RH}$; and $25^{\circ} \mathrm{C}, 32 \% \mathrm{RH}$ corresponding to saturation deficits of $2.57,4.19,9.35$, 11.33, and $15.89 \mathrm{mmHg}(1 \mathrm{mmHg}=133.3 \mathrm{~Pa})$, respectively, as calculated by Randolph and Storey (1999). Survival tests were conducted in the dark. Survival of ticks was recorded after $48 \mathrm{~h}$. This duration was determined so that global survival would tend to be $50 \%$ based on Perret (2002). All ticks, dead or alive, were then stored at $-80^{\circ} \mathrm{C}$ until analysis for Borrelia infection by real-time polymerase chain reaction (PCR) and reverse line blotting (RLB).

Borrelia Infection in Ticks. Before DNA isolation, ticks were soaked in 70\% ethanol and air-dried. Extraction of DNA from ticks was achieved using ammonium hydroxide as described previously (Guy and Stanek 1991, Rijpkema et al. 1996). Negative controls were included during DNA isolation, which consisted of reagents without template DNA.

A real-time PCR amplifying a fragment of the flagellin gene (Schwaiger et al. 2001) was used to detect and quantify Borrelia DNA in all field-collected ticks that were subjected to the survival tests. The strain $B$. afzelii NE1817 was used as quantification standard. Spirochete concentration in culture was evaluated using the Helber chamber. To extract DNA, the culture was washed twice with phosphate-buffered saline $/ \mathrm{MgCl}_{2}$, and the pellet was resuspended in $30 \mu \mathrm{l}$ of water and heated for $15 \mathrm{~min}$ at $100^{\circ} \mathrm{C}$ (Postic et al. 1994). The Borrelia DNA stock was aliquoted at $10^{5}$ spirochetes per $\mu \mathrm{l}$ and stored at $-20^{\circ} \mathrm{C}$. Serial dilutions were made from stored spirochete DNA to obtain six standard solutions with concentrations of Borrelia DNA ranging from 1 to $10^{5}$ copies per $\mu$ l.

The 50- $\mu$ l real-time PCR mixture (Schwaiger et al. 2001 ) consisted of $5 \mu \mathrm{l}$ of $10 \times \mathrm{MgCl}_{2}$ buffer, $2 \mu \mathrm{l}$ of 25 $\mathrm{mM} \mathrm{MgCl} 2,1 \mu \mathrm{l}$ of $10 \mathrm{mM}$ dNTPs, $1 \mu \mathrm{l}$ of $20 \mu \mathrm{M}$ FlaF1A forward primer, $1 \mu \mathrm{l}$ of $20 \mu \mathrm{M}$ FlaRl reverse primer, $1 \mu \mathrm{l}$ of $10 \mu \mathrm{M}$ FlaProbel probe, $0.25 \mu \mathrm{l}$ of HotStar Taq Polymerase (QIAGEN, Basel, Switzerland), $28.75 \mu \mathrm{l}$ of water, and $10 \mu \mathrm{l}$ of the extracted DNA. In each run, one extraction negative control (10 $\mu$; see above), one PCR negative control ( $10 \mu \mathrm{l}$ of water instead of $10 \mu \mathrm{l}$ of the extracted DNA), and three series of the six standards were included.

After an incubation step at $95^{\circ} \mathrm{C}$ for $10 \mathrm{~min}$, the samples were submitted to 45 repeated amplification cycles $\left(95^{\circ} \mathrm{C}\right.$ for $15 \mathrm{~s}, 60^{\circ} \mathrm{C}$ for $1 \mathrm{~min}$ ) (Schwaiger et al. 2001) in an iCycler Optical Module (Bio-Rad Laboratories, Reinach, Switzerland) by using strip optical 


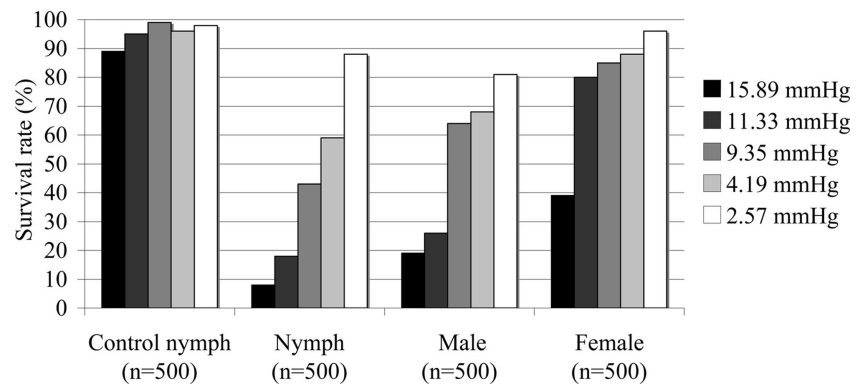

Fig. 1. I. ricinus survival rate after $48 \mathrm{~h}$ spent under thermohygrometric conditions corresponding to values of saturation deficit ranging from 2.57 to $15.89 \mathrm{mmHg}$.

tubes and caps Type F (Life Systems Design, Merenschwand, Switzerland).

PCR and RLB were used to identify the Borrelia species infecting the field-collected ticks. The variable spacer region between two repeated copies of the $23 \mathrm{~S}$ and $5 \mathrm{~S}$ ribosomal genes was amplified with primers 23S-Bor and B-5S-Bor (Alekseev et al. 2001). PCR amplifications were run in a Tgradient Thermocycler 96 (Whatman Biometra, Göttingen, Germany) by using a touchdown PCR program (Burri et al. 2007). Positive and negative controls were included in each PCR. In positive controls, isolates of B. afzelii (NE632), B. burgdorferi s.s. (B31), and B. valaisiana (VS116) replaced DNA samples, whereas water was substituted in negative controls.

For Borrelia identification by RLB, PCR products were hybridized to 15 oligonucleotide probes ( $\mathrm{Ri}$ jpkema et al. 1995, Poupon et al. 2006, Gern et al. 2010) blotted in lines on an activated Biodyne C membrane (Pall Europe Ltd., Portsmouth, United Kingdom) using a Miniblotter 45 (Immunetic, Cambridge, MA). Hybridization was visualized by incubating the membrane with enhanced chemiluminescence detection liquid (Amersham Biosciences Europe, Basel, Switzerland) and by exposing the membrane to X-ray film (Hyperfilm, GE Healthcare, Little Chalfont, Buckinghamshire, United Kingdom).

Statistical Analysis. All statistics were calculated with SPSS Statistics 17.0 for Macintosh (SPSS Inc, Chicago, IL). The relationships between fat content in field-collected nymphs and laboratory-reared nymphs between infection loads in field-collected nymphs, males and females, and between Borrelia genospecies and infection load were evaluated with the Mann-Whitney test. The $\chi^{2}$ test on contingency table was used for assessing the influence of life stages on survival, infection, distribution of Borrelia genospecies, and distribution of infection load categories. Influence of the infection, infection load category, and Borrelia genospecies on survival was assessed by the $\chi^{2}$ test on contingency table. Influence of the thermohygrometric factors and infection load on survival was estimated by binary logistic regressions.

\section{Results}

Fat Content Analysis. Field-collected nymphs contained less fat (8.4\%) than field-collected males (16.2\%; Mann-Whitney test, $P=0)$ or females (13.3\%; MannWhitney test, $P=0.004$ ). In contrast, field-collected males and females did not differ in lipid content. Laboratory-reared nymphs (control) (14.5\%) contained more fat than field-collected nymphs (8.4\%) (MannWhitney test, $P=0$ ).

Survival Tests. Globally, there were differences in survival rates among tick life stages under all five tested conditions. Laboratory-reared nymphs were weakly affected by challenging thermohygrometric conditions showing a survival rate of $95.4 \%$ (477/500). Among field-collected ticks, females displayed the highest survival rate $(77.6 \% ; 388 / 500)$, followed by males $(51.6 \% ; 258 / 500)$ and nymphs $(43.2 \%$; $216 / 500)$.

Temperature and relative humidity had a significant impact on field-collected ticks (binary logistic regression, temperature: nymph, $P=0$; male, $P=0$; female, $P=0$ and binary logistic regression, relative humidity: nymph, $P=0$; male, $P=0$; female, $P=0$ ). In contrast, temperature had no significant influence and relative humidity a light effect on laboratory-reared nymphs (binary logistic regression, $P=0.119$ and $P=0.027$, respectively). More ticks survived when $\mathrm{RH}$ increased and temperature decreased.

SD strongly affected field-collected and laboratoryreared ticks (binary logistic regression: control, $P=$ 0.007; nymph, $P=0$; male, $P=0$; female, $P=0$ ) (Fig. 1 ). Nymphs seemed to suffer from $S D$ at a rate that was proportional to SD increase, whereas in adults some kind of threshold $(\approx 12 \mathrm{mmHg}$ in males and $15 \mathrm{mmHg}$ in females) was observed beyond which survival rate decreased rapidly.

Borrelia Infection in Ticks. Among the 1,500 fieldcollected ticks that were tested for B. burgdorferi s.l. by real-time PCR, 34.8\% $(n=522)$ revealed to be infected: $8.4 \%(n=126)$ were nymphs, $13.2 \%(n=$ $198)$ males, and $13.2 \%(n=198)$ females. Male (39.6\%; $198 / 500)$ and female ticks $(39.6 \% ; 198 / 500)$ were more frequently infected than nymphs $(25.5 \% ; 126 / 500)\left(\chi^{2}\right.$ test, $P=0.001)$. Globally, infection load in these ticks ranged from 1 to 1.2 million spirochetes per tick. Mean spirochete number was highest in females $(52,400$ spirochetes per tick), followed by males (47,600 spi- 
Table 1. Distribution of Borrelia genospecies in field-collected I. ricinus ticks

\begin{tabular}{|c|c|c|c|c|}
\hline Borrelia species ${ }^{a}$ & Nymph $^{b}$ & Male $^{b}$ & Female $^{b}$ & Total $^{c}$ \\
\hline af & $71(14.2)$ & $52(10.4)$ & $45(9)$ & $168(11.2)$ \\
\hline ga & $19(3.8)$ & $59(11.8)$ & $69(13.8)$ & $147(9.8)$ \\
\hline my & $3(0.6)$ & & & $3(0.2)$ \\
\hline ss & $6(1.2)$ & $13(2.6)$ & $18(3.6)$ & $37(2.5)$ \\
\hline va & $11(2.2)$ & $34(6.8)$ & $33(6.6)$ & $78(5.2)$ \\
\hline One species & $110(22)$ & $158(31.6)$ & $165(33)$ & $433(28.9)$ \\
\hline af \& ga & & $3(0.6)$ & $1(0.2)$ & $4(0.3)$ \\
\hline af \& my & $2(0.4)$ & $4(0.8)$ & $4(0.8)$ & $10(0.7)$ \\
\hline af \& ss & $2(0.4)$ & $10(2)$ & $8(1.6)$ & $20(1.3)$ \\
\hline ga \& my & $1(0.2)$ & $2(0.4)$ & & $3(0.2)$ \\
\hline ga \& vs & $8(1.6)$ & $15(3)$ & $17(3.4)$ & $40(2.7)$ \\
\hline ss \& ga & & & $1(0.2)$ & $1(0.1)$ \\
\hline ss \& vs & & $1(0.2)$ & $1(0.2)$ & $2(0.1)$ \\
\hline Two species & $13(2.6)$ & $35(7)$ & $32(6.4)$ & $80(16)$ \\
\hline af \& ga \& vs & & $2(0.4)$ & & $2(0.1)$ \\
\hline af \& my \& ss & & $1(0.2)$ & $1(0.2)$ & $2(0.1)$ \\
\hline af \& ss \& vs & & $1(0.2)$ & & $1(0.1)$ \\
\hline ga \& my \& vs & $1(0.2)$ & & & $1(0.1)$ \\
\hline Three species & $1(0.2)$ & $4(0.8)$ & $1(0.2)$ & $6(0.4)$ \\
\hline
\end{tabular}

${ }^{a}$ af, B. afzelii; ga, B. garinii; my, B. myamotoi; ss, B. burgdorferi sensu stricto; va, B. valaisiana. Values in parentheses are percentages.

${ }^{b} n=500$.

${ }^{c} n=1,500$.

rochetes per tick) and nymphs (18,640 spirochetes per tick). However, the differences were not significant (Mann-Whitney test: nymph-male, $P=0.632$; nymphfemale, $P=0.823$; male-female, $P=0.560$ ).

To compare the infection loads between adults and nymphs, five infection load categories were arbitrarily chosen: very low ( $<180$ spirochetes per tick), low (181-2,100 spirochetes per tick), medium (2,101-16,000 spirochetes per tick), high (16,001160,000 spirochetes per tick), and very high $(>160,000$ spirochetes per tick). Infections in adults fell more often into the high and very high infection load categories (male: 26.8\%, 53/198 and female: $29.3 \%, 58 / 198)$ than infections in nymphs $(16.7 \%$; $21 / 126)$. In fact, the distribution of infection categories was different between adults and nymphs but not between males and females ( $\chi^{2}$ test: nymphmale, $P=0.003$; nymph-female, $P=0.001$; malefemale, $P=0.928$ ).

Identification of Borrelia Species in Ticks. Identification of Borrelia genospecies by RLB was possible in 519/522 ticks. Globally, $84 \%$ (433/519) of these ticks were infected by one Borrelia species, $15 \%$ (80/519) by two species and $1 \%(6 / 519)$ by three species (Table 1). Mixed infections were more frequently observed in adults (male: 19.7\%, 39/198; female: 16.7\%, 33/198) than in nymphs $(11.1 \%, 14 / 126)$.

Five Borrelia species-B. afzelii, B. burgdorferi s.s., B. garinii, B. myamotoi, and B. valaisiana-were identified by RLB (Table 1). Taking into account single and mixed infections, $B$. afzelii was the most frequent genospecies $(n=207 ; 39.7 \%)$, followed by B. garinii $(n=198 ; 37.9 \%)$, B. valaisiana ( $n=124 ; 23.8 \%)$, whereas $B$. burgdorferi s.s. was less frequent $(n=63$; $12.1 \%$ ). The genospecies that was the least often de-

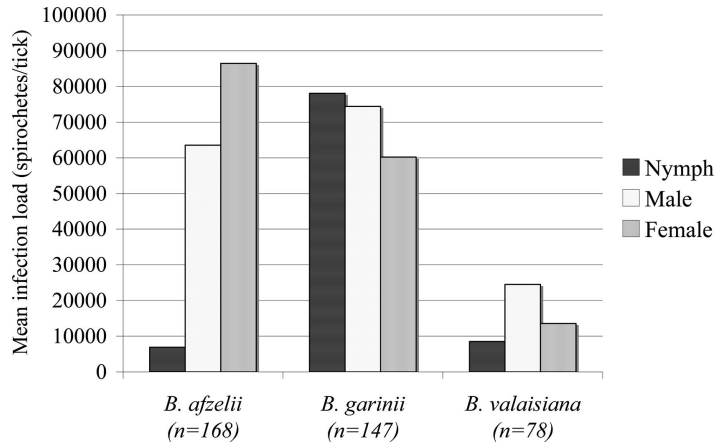

Fig. 2. Mean spirochete load (spirochetes per tick) in nymphal, male and female I. ricinus infected by B. afzelii, $B$. garinii, and B. valaisiana.

tected was B. myamotoi $(n=19 ; 3.6 \%)$. B. bissettii, $B$. lusitaniae, and B. spielmanii, were not observed.

There was no difference in the distribution of $B$. afzelii, B. garinii, and B. valaisiana between males and females ( $\chi^{2}$ test: $B$. garinii, $P=0.416$; $B$. afzelii, $P=$ 0.136 ; B. valaisiana, $P=0.819$ ). However, important dissimilarities between nymphs and adults were observed in the frequency of some Borrelia genospecies. In fact, $B$. garinii and B. valaisiana were more frequent in adults than in nymphs ( $\chi^{2}$ test: nymph-male, $P=0$; nymph-female, $P=0.001$; nymph-male, $P=0.036$; and nymph-female, $P=0.022$, respectively), whereas the contrary was observed for $B$. afzelii ( $\chi^{2}$ test: nymphmale, $P=0$; nymph-female, $P=0)$.

Differences in the infection load were found between tick stages according to Borrelia species (Fig. 2 ). In nymphs, infections by $B$. garinii consisted of more spirochetes $(78,000$ spirochetes per tick) than infections by all other Borrelia species (7,680 spirochetes per tick) (Mann-Whitney test, $P=0.001$ ), whereas this difference was less marked in males (74,400 and 42,800 spirochetes per tick, respectively; Mann-Whitney test, $P=0.028)$ and in females $(60,400$ and 52,400 spirochetes per tick, respectively; MannWhitney test, $P=0.059$ ). In adults, infections by $B$. valaisiana showed less spirochetes (male: 24,520 spirochetes per tick; female: 13,560 spirochetes per tick) than infections by all other Borrelia species (male: 62,800 spirochetes per tick; female: 66,400 spirochetes per tick) (Mann-Whitney test: male, $P=0.007$; female, $P=0.005)$. In nymphs, infections by $B$. valaisiana also displayed less spirochetes than all other Borrelia species, but the difference was not significant (8,520 and 20,880 spirochetes per tick, respectively) (Mann-Whitney test, $P=0.864)$. Finally, it seemed that nymphs infected by B. afzelii (6,880 spirochetes per tick) bore less spirochetes than nymphs infected by other genospecies (41,600 spirochetes per tick) (Mann-Whitney test, $P=0.025)$. Interestingly, the reverse trend was observed in adults, although the difference was not significant (male: 63,600 and 50,400 spirochetes per tick, respectively; female: 86,400 and 44,400 spirochetes per tick, respectively) (MannWhitney test: male, $P=0.368$; female, $P=0.176$ ). 


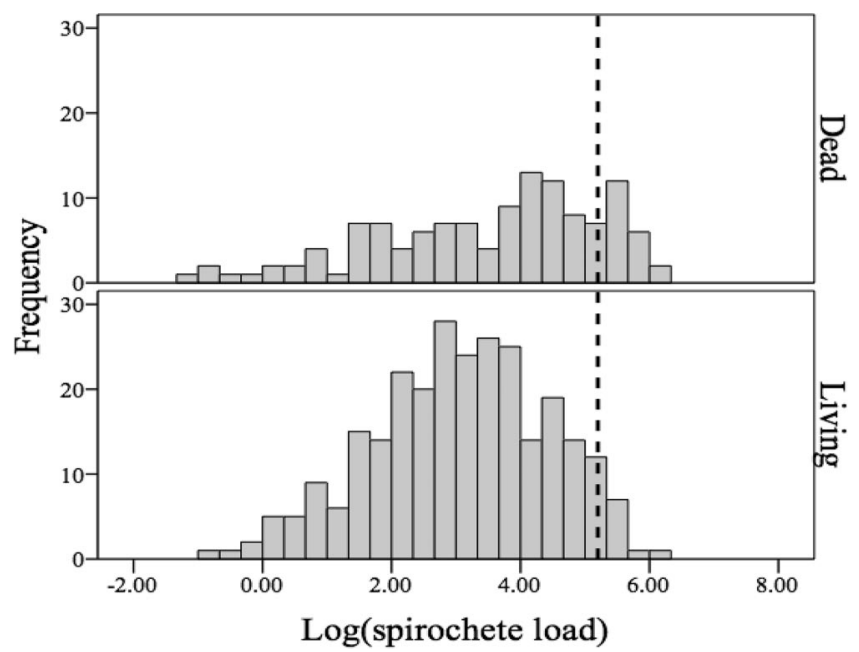

Fig. 3. Distribution of spirochete $\operatorname{load}(\log [$ spirochetes per tick $])$ among dead and living I. ricinus adults. The dashed line lies at 160,000 spirochetes per tick.

Effect of Borrelia Infection on Tick Survival. Infection load had an effect on adult survival (binary logistic regression, $P=0.031$ ) but had no effect on survival of nymphs (binary logistic regression, $P=$ $0.553)$. In adults, survival rate increased as infection load decreased, i.e., a higher infection load negatively influenced adult survival. Specifically, it seemed that infections consisting of $>160,000$ spirochetes per tick had a negative impact on adult survival (Fig. 3). In nymphs, however, high spirochete numbers did not negatively influence survival (data not shown).

Based on the results of the survival tests, we considered that thermohygrometric conditions were challenging for ticks when the survival of ticks was below $70 \%$. Because males and females survived well and were affected by thermohygrometric conditions similarly, both categories were merged together to evaluate the SD values corresponding to a survival rate $<70 \%$. These SD values were $4.19,9.35,11.33$, and $15.89 \mathrm{mmHg}$ in nymphs and 11.33 and $15.89 \mathrm{mmHg}$ in adults. Under these thermohygrometric conditions, infection revealed to have a positive effect on survival of nymphs and adults: $42 / 98$ infected nymphs (42.9\%) and 62/123 infected adults (50.4\%) survived, whereas only 86/302 uninfected nymphs (28.5\%) and 95/251 uninfected adults $(37.9 \%)$ survived ( $\chi^{2}$ test: nymph, $P=0.008$; adult, $P=0.021)$.

The number of genospecies in the tick had no impact on tick survival ( $\chi^{2}$ test, $\left.P=0.589\right)$. However, differences were observed between Borrelia genospecies. Hence, nymphs infected by B. afzelii survived better $(44.1 \%$; $26 / 59)$ than nymphs that were not infected or infected by other Borrelia genospecies $(29.9 \% ; 102 / 341)\left(\chi^{2}\right.$ test, $\left.P=0.031\right)$. The same trend was observed in adults where a higher survival rate $(53.5 \% ; 23 / 43)$ was observed in adults infected by $B$. afzelii than in adults that were not infected or infected by another genospecies $(39.5 \% ; 141 / 357)\left(\chi^{2}\right.$ test, $P=$ $0.057)$.

\section{Discussion}

Fat Content Analysis. In this study, we determined whether B. burgdorferi s.l. influenced I. ricinus survival under thermohygrometric stress. Fat content in I. ricinus was measured to take into account variable energy resources when assessing I. ricinus survival. It was observed that fat content in field-collected females was slightly higher than that in field-collected males and considerably higher than that in field-collected nymphs. These results are in line with what is expected because fat content is a source of energy derived from each bloodmeal (Uspensky 1995). Larvae, which ingest less blood than further developmental stages $(\mathrm{Og}$ den et al. 1998), contain less fat than adults as they molt into nymphs. Likewise, nymphal males that take relatively less blood than nymphal females (Graf 1978, Mbow et al. 1994) molt into adult males with smaller energy resources than adult females.

Survival Tests. Globally, differences in survival were observed among I. ricinus life stages: females survived better $(77.6 \%)$ than males $(51.6 \%)$, which in turn survived better than nymphs $(43.2 \%)$. Lower fat content in nymphs seemed to contribute to lower survival rate. Moreover, adults have a higher water storage capacity due to their size, and their lower surface-to-volume ratio probably allowed them to reduce water loss under dry conditions. Nevertheless the most striking difference in survival lay between laboratory-reared and field-collected nymphs. Although both belonged to the same life stage, the laboratory-reared nymphs survived extremely well (global survival rate, 95.4\%) under any thermohygrometric condition, whereas the field-collected nymphs did not (global survival rate, $43.2 \%$ ). This might be explained by the higher fat content, lack of any kind of stress of laboratory-reared nymphs, or a combination. Hence, they had full and ready-to-use stored energy resources when tests began. 
Ixodes ticks are exceptionally sensitive to temperature and humidity compared with other ticks (Knülle and Rudolph 1982, Sonenshine 1991). More precisely, it has been observed that $I$. ricinus most important climatic feature for survival is a high degree of humidity and that increasing temperature limits survival (MacLeod 1935). Saturation deficit, defined as the drying power of the air (Randolph and Storey 1999), also has revealed to influence $I$. ricinus questing activity (Randolph and Storey 1999; Perret et al. 2000, 2003, 2004) and survival in nature (Perret et al. 2000, 2004; Perret 2002; Burri et al. 2007). Identical observations were made in the current study. Increasing relative humidity influenced positively $I$. ricinus survival, whereas increasing temperature affected it negatively. As a combination of the last two factors, SD had a stronger impact on $I$. ricinus than temperature and relative humidity alone. Hence, survival rate was inversely proportional to SD, i.e., more ticks survived as SD decreased.

The latter was true for all field-collected ticks, although it was more marked in nymphs. This matched fat content results: nymphs that had lower fat content than adults survived less well. Thus, it seems to indicate that available energy resources determine life span, which was described in Randolph and Storey (1999), and that more challenging thermohygrometric conditions increase fat usage. This also applies to laboratory-reared nymphs, although results are not as clear-cut as those of field-collected ticks. It may be due to a stress-free state (fully hydrated, high fat) of colony ticks, leading to a very low death rate $(4.6 \%$; $23 / 500)$ in the survival tests and less straightforward interpretations. However, these results clearly display how different laboratory-reared and field-collected ticks may be.

Borrelia Infection in Ticks. Overall prevalence of infection by Borrelia (34.8\%) was similar to that of another study conducted in the same area $(32.7 \%)$ (Casati et al. 2004). Infection prevalence in adults was higher $(39.6 \%)$ than in nymphs $(25.5 \%)$, as often described in Europe (Rauter and Hartung 2005) and Switzerland (Casati et al. 2004, Jouda et al. 2004). Similarly, adult infections consisted more frequently of $>160,000$ spirochetes than those of nymphs. These differences in infection prevalence and infection load among stages are probably due to the number and size of bloodmeals taken by ticks in their earlier developmental stages (Ogden et al. 1998).

Genospecies prevalence was in line with what had been observed in the same area: B. afzelii $(33.8 \%), B$. burgdorferi s.s. (10.3\%), B. garinii $(32.1 \%)$, B. myamotoi $(3.1 \%)$, and B. valaisiana (20.2\%) (Casati et al. 2004, Jouda et al. 2004, Morán Cadenas et al. 2007b, Gern et al. 2010). The Borrelia genospecies were similarly distributed among males and females. However, B. afzelii prevailed in nymphs compared with adults, and $B$. garinii and B. valaisiana were more frequent in adults compared with nymphs. Different favored hosts for bloodmeal and specific host-parasite association may explain this discrepancy (Humair et al. 1995, 1998,
1999; Humair and Gern 1998; Kurtenbach et al. 1998; Morán Cadenas et al. 2007a).

High loads of spirochetes were usually observed in B. garinii-infected nymphs and adults, and low loads were observed in B. valaisiana-infected mature and immature ticks. B. afzelii was observed in low numbers in nymphs, whereas higher numbers of spirochetes were observed in adults. It has been demonstrated that transmission from I. ricinus to host differs among Borrelia genospecies (Crippa et al. 2002). More precisely, B. afzelii is transmitted to host earlier and more efficiently than $B$. burgdorferi s.s. The latter might be true in the opposite direction, i.e., from host to tick. Nymphs that feed for a longer time than larvae may have ingested more $B$. afzelii spirochetes resulting in adults harboring higher borreliae numbers than nymphs. Thus, early-transmitted spirochetes such as B. afzelii (Crippa et al. 2002) and probably also $B$. garinii according to the present results may be found in higher numbers in ticks than late-transmitted ones such as B. burgdorferi s.s. (Crippa et al. 2002) and supposedly B. valaisiana.

Infection load in questing I. ricinus in Europe has been investigated by Rauter et al. (2002), who conducted a real-time PCR targeting the ospA gene (compared with a fragment of the flagellin gene here). They reported a median of 4,000 spirochetes per tick, which is higher than the one observed in the current study (2,760 per nymph; 1,496 per male; 2,078 per female). However, Rauter et al. (2002) did not discriminate between life stages or between Borrelia genospecies (both factors have been shown to significantly influence infection load).

Effect of Borrelia Infection on Tick Survival. In view of the present results, the hypothesis that infection by B. burgdorferi s.l. enhances I. ricinus survival under thermohygrometric stress conditions is confirmed. However, the phenomenon does not apply in every situation and acts subtly. Its impact varies between life stages, as well as according to infection load. Hence, nymphal survival revealed to be highly positively influenced by infection by Borrelia, whatever the infection load. In contrast, adults seemed to be positively influenced by spirochete colonization up to a threshold of infection load (estimated at 160,000 spirochetes per tick), where an increasing number of borreliae became harmful and the positive effect was reversed to a negative effect. That this deleterious effect of spirochete "overdose" was observed in adults but not in nymphs is probably related to the extremely rare proportion of nymphs bearing $>160,000$ spirochetes per tick $(1.6 \% ; 2 / 126)$ compared with adults $(8.6 \% ; 34 / 396)$. Thus, a potentially negative effect of infection load could not be detected in nymphs due to poor occurrence of very high infection load. Very high Borrelia numbers might trigger I. ricinus immune response (Coleman et al. 1997, Johns et al. 2001, Sonenshine and Hynes 2008) requiring too much energy resources. That would, in turn, reduce energy allowance to other stress sources such as challenging thermohygrometric conditions thereby impairing vector resistance to heat and/or desiccation. 
Although the number of different genospecies infecting the tick did not impact on tick survival, one particular genospecies, B. afzelii, revealed to be positively associated with $I$. ricinus survival, especially in nymphs. Therefore, ticks infected by B. afzelii had an advantage over uninfected ticks or those infected by other Borrelia genospecies when stressed by unfavorable thermohygrometric conditions. Interestingly, it has been observed during the past decade that infection rate by $B$. afzelii in I. ricinus has increased in the Neuchâtel region (L.G., unpublished data) where hot and dry climatic conditions have been shown to have a negative impact on questing tick densities (Perret et al. 2000, 2004; Morán Cadenas et al. 2007b).

Various hypotheses can be put forward to explain the higher survival rate of ticks infected by Borrelia observed in the current study. Although spirochetes in questing ticks are usually limited to the tick midgut, they may be disseminated in other tissues and organs producing a systemic infection in up to $11 \%$ of $I$. ricinus-infected nymphs (Burgdorfer et al. 1989, Lebet and Gern 1994). Hence, we can surmise that spirochetes in the midgut and those that are systemically distributed in the tick might be able to modify the physiology, metabolism, or both, of organs, such as those involved in bloodmeal digestion or water sorption/loss, resulting in optimized blood digestion increasing energy resources or optimized water sorption/retention enhancing water storage, respectively.

However, it is known that infection by Borrelia can change gene expression in ticks as observed in I. scapularis. It was shown that the expression of one gene was selectively increased in salivary glands of ticks infected by B. burgdorferi s.s. during blood feeding (Ramamoorthi et al. 2005). Therefore, it may be hypothesized that spirochetes, which are known to change their gene expression depending on temperature (Ojaimi et al. 2003), might be able to modify tick gene expression under varying temperature conditions as well, inactivating genes governing vector nonvital functions, slowing metabolism down, so that energy resources would essentially be devoted to resisting unfavorable thermohygrometric conditions while maintaining favorable living conditions for spirochetes.

It also has been observed that spirochetes are noninfectious (possibly evading tick immune response) when they are in unfed ticks (Crippa et al. 2002). Moreover it could be suggested that the turning off of infection factors by spirochetes in unfed ticks also might trigger tick metabolism to slow down, explaining the better survival of infected ticks under challenging thermohygrometric conditions.

More specifically, the positive effect of B. afzelii infection on survival of $I$. ricinus nymphs may be due, either to the fact that infections by B. afzelii consisted of low spirochete numbers and rarely reached a threshold beyond which spirochete load was harmful. Or, as mentioned before, it may be linked to B. afzelii being transmitted to the host earlier and more efficiently, compared with B. burgdorferi s.s., suggesting some influence on tick organ physiology (Crippa et al. 2002).

The molecular mechanisms at the tick-pathogen interface that are responsible for the higher survival rate of I. ricinus ticks infected by Borrelia spp. remain to be investigated and additional studies are expected in this subject. According to our results, infection by B. burgdorferi s.l. provides a survival advantage to $I$. ricinus ticks under challenging conditions of temperature and humidity. In fine, both organisms take advantages from each other, hence such a mutualism may have accompanied the entire evolution of Borrelia together with their tick vectors.

\section{Acknowledgments}

We thank Luca Gigandet, and Kangaji Kasongo for help in collecting ticks; Olivier Rais, Francisca Morán Cadenas, Caroline Burri, and Elena Lommano for precious technical help and advice; and Jason Grant for reviewing the English of the manuscript. Results obtained here are part of a project financed by the Swiss National Science Foundation (FN 320030113936 and FN 310030 127064) to L.G. Some of the results are part of the Ph.D. dissertation of C.H.

\section{References Cited}

Aeschlimann, A. 1972. Ixodes ricinus, Linné, 1758 (Ixodoidae, Ixodidae). Essai préliminaire de synthèse sur la biologie de cette espèce en Suisse. Acta Trop. 29: 321-340.

Alekseev, A. N., H. V. Dubinina, I. Van de Pol, and L. M. Schouls. 2001. Identification of Ehrlichia spp. and Borrelia burgdorferi in Ixodes ticks in the Baltic regions of Russia. J. Clin. Microbiol. 39: 2237-2242.

Alekseev, A. N., P. M. Jensen, H. V. Dubinina, L. A. Smirnova, N. A. Makrouchina, and S. D. Zharkov. 2000. Peculiarities of behaviour of taiga (Ixodes persulcatus) and sheep (Ixodes ricinus) ticks (Acarina: Ixodidae) determined by different methods. Folia Parasitol. 47: 147-153.

Burgdorfer, W., S. F. Hayes, and D. Corwin. 1989. Pathophysiology of the Lyme disease spirochete, Borrelia burgdorferi, in ixodid ticks. Rev. Infect. Dis. 11: 1142-1450.

Burri, C., F. Morán Cadenas, V. Douet, J. Moret, and L. Gern. 2007. Ixodes ricinus density and infection prevalence with Borrelia burgdorferi sensu lato along a north-facing altitudinal gradient in the Rhône Valley (Switzerland). Vector-Borne Zoonotic Dis. 7: 50-58.

Casati, S., M. V. Bernasconi, L. Gern, and J. C. Piffaretti. 2004. Diversity within Borrelia burgdorferi sensu lato genospecies in Switzerland by recA gene sequence. FEMS Miocrobiol. Letters 238: 115-123.

Coleman, J. L., J. A. Gebbia, J. Piesman, J. L. Degen, T. H. Bugge, and J. L. Benach. 1997. Plasminogen is required for efficient dissemination of B. burgdorferi in ticks and for enhancement of spirochetemia in mice. Cell 89: 11111119.

Cotté, V., S. Bonnet, M. Cote, and M. Vayssier-Taussat. 2010. Prevalence of five pathogenic agents in questing Ixodes ricinus ticks from western France. Vector-Borne Zoonotic Dis. 10: 1-8.

Crippa, M., O. Rais, and L. Gern. 2002. Investigations on the mode and dynamics of transmission and infectivity of Borrelia burgdorferi sensu stricto and Borrelia afzelii in Ixodes ricinus ticks. Vector-Borne Zoonotic Dis. 2: 3-9.

Crooks, E., and S. E. Randolph. 2006. Walking by Ixodes ricinus ticks: intrinsic and extrinsic factors determine the 
attraction of moisture or host odour. J. Exp. Biol. 209: 2138-2142.

Faulde, M. K., and R. G. Robbins. 2008. Tick infestation and Borrelia burgdorferi s.l. infection-induced increase in host-finding efficacy of female Ixodes ricinus under natural conditions. Exp. Appl. Acarol. 44: 137-145.

Gern, L., V. Douet, Z. Lopez, O. Rais, and F. Morán Cadenas. 2010. Diversity of Borrelia genospecies in Ixodes ricinus ticks in a Lyme borreliosis endemic area in Switzerland identified by using new probes for reverse line blotting. Ticks Tick-Borne Dis. 1: 23-29.

Graf, J.-F. 1978. Copulation, nutrition and oviposition of Ixodes ricinus (Ixodoidae: Ixodidae) Part 1. Bull. Soc. Entomol. Suisse 51: 89-98.

Guy, E. C., and G. Stanek. 1991. Detection of Borrelia burgdorferi in patients with Lyme disease by the polymerase chain reaction. J. Clin. Pathol. 44: 610-611.

Humair, P. F., and L. Gern. 1998. Relationship between Borrelia burgdorferi sensu lato species, red squirrels (Sciurus vulgaris) and Ixodes ricinus in enzootic areas in Switzerland. Acta Trop. 69: 213-227.

Humair, P. F., D. Postic, R. Wallich, and L. Gern. 1998. An avian reservoir (Turdus merula) of the Lyme borreliosis spirochetes. Zentralbl. Bakteriol. 287: 521-538.

Humair, P. F., O. Rais, and L. Gern. 1999. Transmission of Borrelia afzelii from Apodemus mice and Clethrionomys voles to Ixodes ricinus: differential transmission pattern and overwintering maintenance. Parasitology 118: 33-42.

Humair, P. F., O. Péter, R. Wallich, and L. Gern. 1995. Strain variation of Lyme disease spirochetes isolated from Ixodes ricinus ticks and rodents collected in two endemic areas in Switzerland. J. Med. Entomol. 32: 433-438.

Johns, R., J. Ohnishi, A. Broadwater, D. E. Sonenshine, A. M. de Silva, and W. L. Hynes. 2001. Contrasts in tick innate immune responses to Borrelia burgdorferi challenge: immunotolerance in Ixodes scapularis versus immunocompetence in Dermacentor variabilis (Acari: Ixodidae). J. Med. Entomol. 38: 99-107.

Jouda, F., J.-L. Perret, and L. Gern. 2004. Ixodes ricinus density, and distribution and prevalence of Borrelia burgdorferi sensu lato infection along an altitudinal gradient. J. Med. Entomol. 41: 162-169.

Knülle, W., and D. Rudolph. 1982. Humidity relationships and water balance of ticks, pp. 43-70. In F. D. Obenchain and R. Galun (eds.), Physiology of ticks. Pergamon, Oxford, United Kingdom.

Kurtenbach, K., M. Peacy, S.G.T. Rijpkema, A. N. Hoodless, P. A. Nuttall, and S. E. Randolph. 1998. Differential transmission of the genospecies of Borrelia burgdorferi sensu lato by game birds and small rodents in England. Appl. Environ. Microbiol. 64: 1169-1174.

Lebet, N., and L. Gern. 1994. Histological examination of Borrelia burgdorferi infections in unfed Ixodes ricinus nymphs. Exp. Appl. Acarol. 18: 177-183.

Lees, A. D. 1946. Water balance in Ixodes ricinus L. and certain other species of ticks. Parasitology 37: 1-20.

Lefcort, H., and L. A. Durden. 1996. The effect of infection with Lyme disease spirochetes (Borrelia burgdorferi) on the phototaxis, activity, and questing height of the tick vector Ixodes scapularis. Parasitology 113: 97-103.

MacLeod, J. 1935. Ixodes ricinus in relation to its physical environment. II. The factors governing survival and activity. Parasitology 27: 123-144.

Margos, G., S. A. Vollmer, M. Cornet, M. Garnier, V. Fingerle, B. Wilske, A. Bormane, L. Vitorino, M. CollaresPereira, M. Drancourt, et al. 2009. A new Borrelia species defined by multilocus sequence analysis of housekeeping genes. Appl. Environ. Microbiol. 75: 5410-5416.
Mather, T. N., D. C. Duffy, and S. R. Campbell. 1993. An unexpected result from burning vegetation to reduce Lyme disease transmission risks. J. Med. Entomol. 30: 642-645.

Mbow, M. L., M. Christe, B. Rutti, and M. Brossard. 1994. Absence of acquired resistance to nymphal Ixodes ricinus ticks in BALB/C mice developing cutaneous reactions. J. Parasitol. 80: 81-87.

Morán Cadenas, F., O. Rais, P.-F. Humair, V. Douet, J. Moret, and L. Gern. 2007a. Identification of host bloodmeal source and Borrelia burgdorferi sensu lato in field-collected Ixodes ricinus ticks in Chaumont (Switzerland). J. Med. Entomol. 44: 1109-1117.

Morán Cadenas, F., O. Rais, F. Jouda, V. Douet, P.-F. Humair, J. Moret, and L. Gern. 2007b. Phenology of Ixodes ricinus and infection with Borrelia burgdorferi sensu lato along a north- and south-facing altitudinal gradient on Chaumont Mountain, Switzerland. J. Med. Entomol. 44: 683-693.

Naumov, R. L. 1999. The questing activity of the taiga tick Ixodes persulcatus infected with borreliae. Parazitologiya 33: 251-256.

Naumov, R. L. 2003. The longevity of the sheep and taiga ticks (Ixodidae) infected and uninfected with borreliae of the burgdorferi group. Parazitologiya 37: 527-532.

Ogden, N. H., K. Kurtenbach, and P. A. Nuttall. 1998. Interstadial and infestation level-dependent variation in the transmission efficiency of Borrelia burgdorferi from mice to Ixodes ricinus ticks. Exp. Appl. Acarol. 22: 367-372.

Ojaimi, C., C. Brooks, S. Casjens, P. Rosa, A. Elias, A. Barbour, A. Jasinskas, J. Benach, L. Katona, J. Radolf, M. Casimano, J. Skare, K. Swingle, D. Atkins, and I. Schwartz. 2003. Profiling of temperature-induced changes in Borrelia burgdorferi gene expression by using whole genome arrays. Infect. Immun. 71: 1689-1705.

Perret, J.-L. 2002. Computer-assisted laboratory and field studies of the host-finding behaviour of the tick Ixodes ricinus (Acarina: Ixodidae): ecological implications of climate and light. Ph.D. dissertation, Université de Neuchâtel, Neuchâtel, Switzerland.

Perret, J.-L., E. Guigoz, O. Rais, and L. Gern. 2000. Influence of saturation deficit and temperature on Ixodes ricinus tick questing activity in Lyme borreliosis-endemic area (Switzerland). Parasitol. Res. 86: 554-557.

Perret, J.-L., P. M. Guerin, P. A. Diehl, M. Vlimant, and L. Gern. 2003. Darkness induces mobility, and saturation deficit limits questing duration, in the tick Ixodes ricinus. J. Exp. Biol. 206: 1809-1815.

Perret, J.-L., O. Rais, and L. Gern. 2004. Influence of climate on the proportion of Ixodes ricinus nymphs and adults questing in a tick population. J. Med. Entomol. 41: 361365.

Postic, D., M. V. Assous, P.A.D. Grimont, and G. Baranton. 1994. Diversity of Borrelia burgdorferi sensu lato evidenced by restriction fragment length polymorphism of $r r f(5 S)-r r l(23 S)$ intergenic spacer amplicons. Int. J. Syst. Bacteriol. 44: 743-752.

Poupon, M.-A., E. Lommano, P.-F. Humair, V. Douet, O. Rais, M. Schaad, L. Jenni, and L. Gern. 2006. Prevalence of Borrelia burgdorferi sensu lato in ticks collected from migratory birds in Switzerland. Appl. Environ. Microbiol. 72: 976-979.

Ramamoorthi, N., S. Narasimhan, U. Pal, F. Bao, X. F. Yang, D. Fish, J. Anguita, M. V. Norgard, F. S. Kantor, J. F. Anderson, et al. 2005. The Lyme disease agent exploits a tick protein to infect the mammalian host. Nature 436 573-577. 
Randolph, S. E., and K. Storey. 1999. Impact of microclimate on immature tick-rodent interactions (Acari: Ixodidae): implications for parasite transmission. J. Med. Entomol. 36: 741-748.

Rauter, C., and T. Hartung. 2005. Prevalence of Borrelia burgdorferi sensu lato genospecies in Ixodes ricinus ticks in Europe: a metaanalysis. Appl. Environ. Microbiol. 71: 7203-7216.

Rauter, C., R. Oehme, I. Diterich, M. Engele, and T. Hartung. 2002. Distribution of clinically relevant Borrelia genospecies in ticks assessed by a novel, single-run, real-time PCR. J. Clin. Microbiol. 40: 36-43.

Rijpkema, S.G.T., D. Golubic, M.J.C.H. Molkenboer, N. Verbeek-De Kruif, and J.F.P. Schellekens. 1996. Identification of four genomic groups of Borrelia burgdorferi sensu lato in Ixodes ricinus ticks collected in a Lyme borreliosis endemic region of northern Croatia. Exp. Appl. Acarol. 20: $23-30$.

Rijpkema, S.G.T., M.J.C.H. Molkenboer, L. M. Schouls, F. Jongejan, and J.F.P. Schellekens. 1995. Simultaneous detection and genotyping of three genomic groups of Borrelia burgdorferi sensu lato in Dutch Ixodes ricinus ticks by characterization of the amplified intergenic spacer region between $5 \mathrm{~S}$ and $23 \mathrm{~S}$ rRNA genes. J. Clin. Microbiol. 33: 3091-3095.

Schwaiger, M., O. Péter, and P. Cassinotti. 2001. Routine diagnosis of Borrelia burgdorferi (sensu lato) infections using real-time PCR assay. Clin. Microbiol. Infect. 7: 461469.

Sonenshine, D. E. 1991. Biology of ticks. Oxford University Press, New York.

Sonenshine, D. E., and W. L. Hynes. 2008. Molecular characterization and related aspects of the innate immune response in ticks. Front. Biosci. 13: 7046-7063.

Uspensky, I. 1995. Physiological age of ixodid ticks: aspects of its determination and application. J. Med. Entomol. 32: $751-764$.

Uspensky, I., Y. V. Kovalevskii, and E. I. Korenberg. 2006. Physiological age of field-collected female taiga ticks, Ixodes persulcatus (Acari: Ixodidae), and their infection with Borrelia burgdorferi sensu lato. Exp. Appl. Acarol. 38: 201-209.

Winston, P. W., and B. H. Bates. 1960. Saturated solutions for the control of humidity in biological research. Ecology 41: 232-237.

Received 26 April 2010; accepted 13 July 2010. 